PROCEEDINGS OF THE

AMERICAN MATHEMATICAL SOCIETY

Volume 138, Number 6, June 2010, Pages 2125-2134

S 0002-9939(10)10276-7

Article electronically published on February 3, 2010

\title{
APPLICATIONS OF NEW GERONIMUS TYPE IDENTITIES FOR REAL ORTHOGONAL POLYNOMIALS
}

\author{
D. S. LUBINSKY
}

(Communicated by Walter Van Assche)

\begin{abstract}
Let $\mu$ be a positive measure on the real line, with associated orthogonal polynomials $\left\{p_{n}\right\}$. Let $\operatorname{Im} a \neq 0$. Then there is an explicit constant $c_{n}$ such that for all polynomials $P$ of degree at most $2 n-2$,

$$
c_{n} \int_{-\infty}^{\infty} \frac{P(t)}{\left|p_{n}(a) p_{n-1}(t)-p_{n-1}(a) p_{n}(t)\right|^{2}} d t=\int P d \mu .
$$

In this paper, we provide a self-contained proof of this identity. Moreover, we apply the formula to deduce a weak convergence result and a discrepancy estimate, and also to establish a Gauss quadrature associated with $\mu$ with nodes at the zeros of $p_{n}(a) p_{n-1}(t)-p_{n-1}(a) p_{n}(t)$.
\end{abstract}

\section{INTRODUCTION}

Let $\mu$ be a positive measure on the real line with infinitely many points in its support and $\int x^{j} d \mu(x)$ finite for $j=0,1,2, \ldots$ Then we may define orthonormal polynomials

$$
p_{n}(x)=\gamma_{n} x^{n}+\cdots, \quad \gamma_{n}>0,
$$

satisfying

$$
\int_{-\infty}^{\infty} p_{n} p_{m} d \mu=\delta_{m n}
$$

In analysis and applications of orthogonal polynomials, the reproducing kernel

$$
K_{n}(x, y)=\sum_{j=0}^{n-1} p_{j}(x) p_{j}(y)
$$

plays a key role. The Christoffel-Darboux formula asserts that

$$
K_{n}(x, y)=\frac{\gamma_{n-1}}{\gamma_{n}} \frac{p_{n}(x) p_{n-1}(y)-p_{n-1}(x) p_{n}(y)}{x-y} .
$$

We shall also use the notation

$$
L_{n}(x, y)=(x-y) K_{n}(x, y)=\frac{\gamma_{n-1}}{\gamma_{n}}\left(p_{n}(x) p_{n-1}(y)-p_{n-1}(x) p_{n}(y)\right)
$$

Received by the editors August 17, 2009, and, in revised form, October 22, 2009.

2010 Mathematics Subject Classification. Primary 42C05; Secondary 41A17, 41A10, 41A55.

Key words and phrases. Orthogonal polynomials on the real line, Geronimus formula, discrepancy, weak convergence, Gauss quadrature.

This research was supported by NSF grant DMS0700427 and U.S.-Israel BSF grant 2004353. 
and, for non-real $a$,

$$
E_{n, a}(z)=\sqrt{\frac{2 \pi}{\left|L_{n}(a, \bar{a})\right|}} L_{n}(\bar{a}, z) .
$$

In a recent paper [4, we used the theory of de Branges spaces [1] to show that for $\operatorname{Im} a>0$ and all polynomials $P$ of degree $\leq 2 n-2$, we have

$$
\int_{-\infty}^{\infty} \frac{P(t)}{\left|E_{n, a}(t)\right|^{2}} d t=\int P d \mu
$$

This may be regarded as an analogue of Geronimus's formula for the unit circle, where instead of $E_{n, a}$ we have a multiple of the orthonormal polynomial on the unit circle in the denominator (see [2, Thm. V.2.2, p. 198] and [5, pp. 95 and 955]). The name Geronimus's formula is not universal; some talk of continuous analogues of quadrature, or Bernstein-Szegö approximations. There is an earlier real line analogue, rediscovered by Barry Simon [6. Theorem 2.1, p. 5], namely

$$
\frac{1}{\pi} \int_{-\infty}^{\infty} \frac{P(t)}{\left(\frac{\gamma_{n-1}}{\gamma_{n}}\right)^{2} p_{n}^{2}(t)+p_{n-1}^{2}(t)} d t=\int P d \mu .
$$

Simon calls this a real line orthogonal polynomial analogue of Carmona's formula and refers to earlier work of Krutikov and Remling [3. This latter formula seems to be a special case of (1.3) with $\left(p_{n-1} / p_{n}\right)(\bar{a})= \pm i \gamma_{n-1} / \gamma_{n}$. As far as the author is aware, (1.3) is new. At least, the author could not find it in a search of the orthogonal polynomial and orthogonal rational function literature.

In this paper, we present a self-contained proof of (1.3) and deduce results on weak convergence, discrepancy estimates, and a Gauss quadrature type formula with complex nodes. Recall that $\mu$ is said to be determinate if the moment problem

$$
\int x^{j} d \nu(x)=\int x^{j} d \mu(x), \quad j=0,1,2, \ldots,
$$

has the unique solution $\nu=\mu$ from the class of positive measures. We also say that a function $f$ has polynomial growth at $\infty$ if for some $L>0$ and for large enough $|x|$,

We shall prove:

$$
|f(x)| \leq|x|^{L} .
$$

Theorem 1.1. Let $\mu$ be a positive measure on the real line with all finite power moments and let $\mu$ be determinate. Let $\left\{a_{n}\right\}$ be a sequence of complex numbers with non-zero imaginary part. Then for all functions $f: \mathbb{R} \rightarrow \mathbb{R}$ having polynomial growth at $\infty$ that are Riemann-Stieltjes integrable with respect to $\mu$, we have

$$
\lim _{n \rightarrow \infty} \int_{-\infty}^{\infty} f(x) \frac{d x}{\left|E_{n, a_{n}}(x)^{2}\right|}=\int f d \mu .
$$

Of course, if $f$ is continuous on the real line, it will be locally Riemann-Stieltjes integrable with respect to $\mu$. Simon [6] noted the weak convergence involving his Carmona type formula. When $\mu$ is indeterminate, the weak convergence will fail, since then $E_{n, a}$ has a finite limiting value in the plane. In this case, the limit (1.4) probably holds only for a limited class of entire functions.

One consequence of the weak convergence is that $1 /\left|E_{n, a}\right|^{2} \rightarrow 0$ outside the support, in some sense, yielding information on the behavior of $K_{n}$ : 
Corollary 1.2. Assume the hypotheses of Theorem 1.1.

(a) Let $J$ be a closed subset of $\mathbb{R} \backslash \operatorname{supp}[\mu]$. Then

$$
\lim _{n \rightarrow \infty}\left|\operatorname{Im} a_{n}\right| K_{n}\left(a_{n}, \bar{a}_{n}\right) \int_{J} \frac{d t}{\left(t^{2}+\left|a_{n}\right|^{2}\right)\left|K_{n}\left(t, a_{n}\right)\right|^{2}}=0 .
$$

(b) Assume, in addition, that $\operatorname{supp}[\mu]$ is compact and that $J$ is a compact set disjoint from $\operatorname{supp}[\mu]$. Then

$$
\limsup _{n \rightarrow \infty}\left\{\frac{\left|\operatorname{Im} a_{n}\right|}{1+\left|a_{n}\right|^{2}} K_{n}\left(a_{n}, \bar{a}_{n}\right) \int_{J} \frac{d t}{\left|K_{n}\left(t, a_{n}\right)\right|^{2}}\right\}^{1 / n}<1 .
$$

We can also prove a discrepancy type estimate for the measure $\frac{d t}{\left|E_{n, a}(t)\right|^{2}}-d \mu(t)$. The main tool here is the Markov-Stieltjes inequalities, and the formulation involves the Christoffel function

$$
\lambda_{n}(x)=\frac{1}{K_{n}(x, x)}=\inf _{\operatorname{deg}(P) \leq n-1} \frac{\int P^{2} d \mu}{P^{2}(x)} .
$$

Theorem 1.3. Assume the hypotheses of Theorem 1.1. Let $c, d \in \operatorname{supp}[\mu]$ and $\varepsilon>0$. Then for large enough $n$, we have

$$
\sup _{x \in[c, d]}\left|\int_{-\infty}^{x}\left(\frac{d t}{\left|E_{n, a_{n}}(t)\right|^{2}}-d \mu(t)\right)\right| \leq 3 \sup _{x \in[c-\varepsilon, d+\varepsilon]} \lambda_{n}(x) .
$$

We note that the same estimate (1.7) holds when $\frac{d t}{\left|E_{n, a_{n}}(t)\right|^{2}}$ is replaced by any positive measure sharing the same first $2 n-2$ power moments with $\mu$. Another consequence of (1.3) is a Gauss type quadrature formula with complex nodes. Recall that if we fix real $\xi$, then $L_{n}(t, \xi)$ has $n$ or $n-1$ real zeros $\left\{t_{j n}\right\}$, one of which is $\xi$. There is an associated Gauss quadrature rule [2, Thm. I.3.2, p. 21]:

$$
\sum_{j} \lambda_{n}\left(t_{j n}\right) P\left(t_{j n}\right)=\int P d \mu
$$

valid for all polynomials $P$ of degree $\leq 2 n-2$. The classical Gauss quadrature, involving the zeros $\left\{x_{j n}\right\}$ of $p_{n}$, is the case where $\xi$ is a zero of $p_{n}$. By using elementary properties of the Poisson kernel, one can show that if we let $\operatorname{Im} a$ approach 0 in (1.3), then we obtain this last quadrature formula. In general, when $a$ has non-zero imaginary part, one obtains an analogue of (1.8) with complex nodes. In the formulation, we need the Schwarz reflection of a function $g$,

$$
g^{*}(z)=\overline{g(\bar{z})} .
$$

Theorem 1.4. Let $\mu$ be a positive measure on the real line with at least $n+1$ points in its support and the first $2 n$ finite power moments. Let $a \in \mathbb{C} \backslash \mathbb{R}$ and $\left\{z_{j}\right\}_{j=1}^{n}$ denote the zeros of $L_{n}(a, \cdot)$. Assume they are simple, and let

$$
\lambda_{j}=\frac{2 \pi i}{E_{n, a}\left(z_{j}\right) E_{n, a}^{* \prime}\left(z_{j}\right)}, \quad 1 \leq j \leq n .
$$

Then for all polynomials $P$ of degree at most $2 n-2$,

$$
\sum_{j=1}^{n} \lambda_{j} P\left(z_{j}\right)=\int P d \mu
$$


We note that it is possible, for some finitely many exceptional choices of $a$, that $E_{n, a}$ has multiple zeros; see the remark after Lemma 2.2. In this case, the quadrature involves derivatives of $P$ at the multiple zeros. We note too that as $\operatorname{Im} a \rightarrow 0$, this last formula reduces to (1.8).

\section{Proof of (1.3)}

The proof uses similar ideas to those in [4], but is easier to follow because it is self contained and avoids use of the de Branges theory. Throughout, we assume the hypotheses of Theorem 1.1.

Theorem 2.1. Let $a \in \mathbb{C} \backslash \mathbb{R}$. For polynomials $R$ of degree at most $2 n-2$,

$$
\int_{-\infty}^{\infty} \frac{R(t)}{\left|E_{n, a}(t)\right|^{2}} d t=\int R d \mu
$$

Recall that $L_{n}(z, v)=(z-v) K_{n}(z, v)$ and the notation (1.9) for the Schwarz reflection.

\section{Lemma 2.2.}

(a) For all complex $\alpha, \beta, z, v$,

$$
L_{n}(z, v) L_{n}(\alpha, \beta)=L_{n}(\alpha, z) L_{n}(\beta, v)-L_{n}(\beta, z) L_{n}(\alpha, v) .
$$

(b) Let $\operatorname{Im} a>0$. Then

$$
K_{n}(z, v)=\frac{i}{2 \pi} \frac{E_{n, a}(z) E_{n, a}^{*}(v)-E_{n, a}^{*}(z) E_{n, a}(v)}{z-v} .
$$

(c) If $\operatorname{Im} a>0$, all zeros of $E_{n, a}$ are in the lower half plane.

Proof.

(a) Just substitute the definition (1.1) of $L_{n}$ into the right-hand side of (2.2), then multiply out, cancel factors, and refactorize.

(b) The identity (2.2) with $\alpha=a$ and $\beta=\bar{a}$ gives

$$
L_{n}(z, v) L_{n}(a, \bar{a})=L_{n}(a, z) L_{n}(\bar{a}, v)-L_{n}(\bar{a}, z) L_{n}(a, v) .
$$

Since $L_{n}(z, v)$ has real coefficients as a polynomial in $z, v$ and

$$
L_{n}(a, \bar{a})=2 i \operatorname{Im} a K_{n}(a, \bar{a})=i\left|L_{n}(a, \bar{a})\right|,
$$

we obtain

$$
K_{n}(z, v)=\frac{i}{\left|L_{n}(a, \bar{a})\right|} \frac{L_{n}(\bar{a}, z) \overline{L_{n}(\bar{a}, \bar{v})}-\overline{L_{n}(\bar{a}, \bar{z})} L_{n}(\bar{a}, v)}{z-v},
$$

and (2.3) follows on taking account of (1.2).

(c) It suffices to show that $K_{n}(\bar{a}, \cdot)$ has all its zeros in the lower half plane, since $E_{n, a}$ is a multiple of $(\cdot-\bar{a}) K_{n}(\bar{a}, \cdot)$. In turn, in view of the ChristoffelDarboux formula and the fact that $p_{n-1}$ and $p_{n}$ have real zeros, it suffices to show that

$$
\frac{p_{n-1}(z)}{p_{n}(z)}-\frac{p_{n-1}(\bar{a})}{p_{n}(\bar{a})}
$$

cannot vanish for $\operatorname{Im} z \geq 0$. By the Lagrange interpolation formula at the zeros $\left\{x_{j n}\right\}$ of $p_{n}$, or by partial fraction decomposition,

$$
\frac{p_{n-1}(z)}{p_{n}(z)}=\sum_{j=1}^{n} \frac{p_{n-1}\left(x_{j n}\right)}{p_{n}^{\prime}\left(x_{j n}\right)} \frac{1}{z-x_{j n}} .
$$


Applying l'Hospital's rule to the Christoffel-Darboux formula gives

$$
K_{n}(x, x)=\frac{\gamma_{n-1}}{\gamma_{n}}\left(p_{n}^{\prime}(x) p_{n-1}(x)-p_{n-1}^{\prime}(x) p_{n}(x)\right),
$$

and in particular,

$$
K_{n}\left(x_{j n}, x_{j n}\right)=\frac{\gamma_{n-1}}{\gamma_{n}} p_{n}^{\prime}\left(x_{j n}\right) p_{n-1}\left(x_{j n}\right) .
$$

Thus

$$
\frac{p_{n-1}(z)}{p_{n}(z)}=\frac{\gamma_{n-1}}{\gamma_{n}} \sum_{j=1}^{n} \frac{p_{n-1}^{2}\left(x_{j n}\right)}{K_{n}\left(x_{j n}, x_{j n}\right)} \frac{1}{z-x_{j n}}
$$

so

$$
\operatorname{Im}\left(\frac{p_{n-1}(z)}{p_{n}(z)}\right)=-(\operatorname{Im} z) \frac{\gamma_{n-1}}{\gamma_{n}} \sum_{j=1}^{n} \frac{p_{n-1}^{2}\left(x_{j n}\right)}{K_{n}\left(x_{j n}, x_{j n}\right)} \frac{1}{\left|z-x_{j n}\right|^{2}} .
$$

In particular, for $\operatorname{Im} z>0, \operatorname{Im}\left(\frac{p_{n-1}(z)}{p_{n}(z)}\right)<0$; at the same time, $\operatorname{Im} \bar{a}<0$, $\operatorname{Im}\left(\frac{p_{n-1}(\bar{a})}{p_{n}(\bar{a})}\right)>0$, so $\frac{p_{n-1}(z)}{p_{n}(z)}-\frac{p_{n-1}(\bar{a})}{p_{n}(\bar{a})}$ cannot be zero.

Remark. It is possible for $E_{n, a}$ to have multiple zeros. Indeed, we see that this occurs iff both

$$
\begin{aligned}
& p_{n}(z) p_{n-1}(\bar{a})-p_{n-1}(z) p_{n}(\bar{a})=0 \text { and } \\
& p_{n}^{\prime}(z) p_{n-1}(\bar{a})-p_{n-1}^{\prime}(z) p_{n}(\bar{a})=0 .
\end{aligned}
$$

These latter two relations are equivalent to

$$
p_{n}^{\prime}(z) p_{n-1}(z)-p_{n-1}^{\prime}(z) p_{n}(z)=0 \text { and } \frac{p_{n-1}}{p_{n}}(z)=\frac{p_{n-1}}{p_{n}}(\bar{a}) .
$$

Let us choose a $z$ that is one of the $n-1$ zeros of $p_{n}^{\prime} p_{n-1}-p_{n-1}^{\prime} p_{n}$ in the lower half plane. (It is easily seen by differentiating (2.5) that there are none on the real line, and of course, they occur in conjugate pairs.) Then let us choose $a$ with $\operatorname{Im} a>0$ such that

$$
\frac{p_{n-1}}{p_{n}}(z)=\frac{p_{n-1}}{p_{n}}(\bar{a}) .
$$

There are $n$ choices for $a$, counting multiplicity. For this choice of $a, E_{n, a}$ will have at least a double zero at $z$. Of course, there are only finitely many such exceptional $a$.

Proof of Theorem 2.1. We shall assume $\operatorname{Im} a>0$. The case where $\operatorname{Im} a<0$ follows by taking conjugates. We first prove the reproducing kernel relation

$$
P(z)=\int_{-\infty}^{\infty} \frac{P(t) K_{n}(t, z)}{\left|E_{n, a}(t)\right|^{2}} d t=\int_{-\infty}^{\infty} \frac{P(t) K_{n}(t, z)}{E_{n, a}(t) E_{n, a}^{*}(t)} d t .
$$


Here $z$ is any complex number, and $P$ is any polynomial of degree at most $n-1$. Let us assume first that $\operatorname{Im} z>0$. From the formula (2.3) for $K_{n}$, we see that

$$
\begin{aligned}
& \int_{-\infty}^{\infty} \frac{P(t) K_{n}(t, z)}{E_{n, a}(t) E_{n, a}^{*}(t)} d t \\
& \quad=\frac{i}{2 \pi}\left(E_{n, a}^{*}(z) \int_{-\infty}^{\infty} \frac{P(t)}{E_{n, a}^{*}(t)(t-z)} d t-E_{n, a}(z) \int_{-\infty}^{\infty} \frac{P(t)}{E_{n, a}(t)(t-z)} d t\right) .
\end{aligned}
$$

Recall that $E_{n, a}$ has all its zeros in the lower half plane, so $E_{n, a}^{*}$ has all its zeros in the upper half plane. Then the integrand $\frac{P(t)}{E_{n, a}^{*}(t)(t-z)}$ in the first integral is analytic as a function of $t$ in the closed lower half plane, and is $O\left(|t|^{-2}\right)$ as $|t| \rightarrow \infty$. By the residue theorem, or Cauchy's integral theorem, the first integral is 0 . Next, the integrand $\frac{P(t)}{E_{n, a}(t)(t-z)}$ in the second integral is analytic as a function of $t$ in the closed upper half plane, except for a simple pole at $z$ (unless $P(z)=0$ ), and is $O\left(|t|^{-2}\right)$ as $|t| \rightarrow \infty$. The residue theorem shows that

$$
\int_{-\infty}^{\infty} \frac{P(t)}{E_{n, a}(t)(t-z)} d t=2 \pi i \frac{P(z)}{E_{n, a}(z)} .
$$

Substituting this into (2.7) gives (2.6) for $\operatorname{Im} z>0$. As both sides of (2.6) are polynomials in $z$, analytic continuation gives that it holds for all $z$.

Now we can prove (2.1). We can write $R=P S$ where both $P$ and $S$ are polynomials of degree $\leq n-1$. We multiply the identity in (2.6) by $S$ and then integrate with respect to $\mu$. We obtain

$$
\begin{aligned}
\int R d \mu & =\int(P S)(z) d \mu(z) \\
& =\int S(z)\left[\int_{-\infty}^{\infty} P(t) \frac{K_{n}(t, z)}{\left|E_{n, a}(t)\right|^{2}} d t\right] d \mu(z) \\
& =\int_{-\infty}^{\infty} P(t) \frac{1}{\left|E_{n, a}(t)\right|^{2}}\left[\int S(z) K_{n}(t, z) d \mu(z)\right] d t \\
& =\int_{-\infty}^{\infty} P(t) \frac{1}{\left|E_{n, a}(t)\right|^{2}} S(t) d t \\
& =\int_{-\infty}^{\infty} \frac{R}{\left|E_{n, a}\right|^{2}} .
\end{aligned}
$$

Here, we have used the reproducing kernel formula for the measure $\mu$. Moreover, the interchange of integrals is justified by absolute convergence of all integrals involved.

\section{Weak convergence, discrepancy, and Gauss quadrature}

Proof of Theorem 1.1. Let $f$ be Riemann-Stieltjes integrable with respect to $\mu$ and of polynomial growth at $\infty$, and let $\varepsilon>0$. Since $\mu$ is determinate, there exist upper and lower polynomials $P_{u}$ and $P_{\ell}$ such that

$$
P_{\ell} \leq f \leq P_{u} \text { in }(-\infty, \infty)
$$


and

$$
\int\left(P_{u}-P_{\ell}\right) d \mu<\varepsilon
$$

See, for example, [2, Theorem 3.3, p. 73]. Then for $n$ so large that $2 n-2$ exceeds the degree of $P_{u}$ and $P_{\ell},(1.3)$ gives

$$
\begin{aligned}
\int_{-\infty}^{\infty} \frac{f}{\left|E_{n, a_{n}}\right|^{2}}-\int f d \mu & =\int_{-\infty}^{\infty} \frac{f-P_{\ell}}{\left|E_{n, a_{n}}\right|^{2}}-\int\left(f-P_{\ell}\right) d \mu \\
& \leq \int_{-\infty}^{\infty} \frac{P_{u}-P_{\ell}}{\left|E_{n, a_{n}}\right|^{2}}-0 \\
& =\int\left(P_{u}-P_{\ell}\right) d \mu<\varepsilon .
\end{aligned}
$$

Similarly, for large enough $n$,

$$
\begin{aligned}
\int_{-\infty}^{\infty} \frac{f}{\left|E_{n, a_{n}}\right|^{2}}-\int f d \mu & =\int_{-\infty}^{\infty} \frac{f-P_{u}}{\left|E_{n, a_{n}}\right|^{2}}+\int\left(P_{u}-f\right) d \mu \\
& \geq \int_{-\infty}^{\infty} \frac{P_{\ell}-P_{u}}{\left|E_{n, a_{n}}\right|^{2}}+0 \\
& =\int\left(P_{\ell}-P_{u}\right) d \mu>-\varepsilon .
\end{aligned}
$$

Proof of Corollary 1.2. (a) For all real $x$, let

$$
f(x)=\frac{\operatorname{dist}(x, \operatorname{supp}[\mu])}{1+\operatorname{dist}(x, \operatorname{supp}[\mu])} \text {. }
$$

Here dist is the usual Euclidean distance between a point and a set. Then $f$ is continuous, $0 \leq f \leq 1$ for all real $x, f=0$ in $\operatorname{supp}[\mu]$, and for $x \in J$,

$$
f(x) \geq \frac{\operatorname{dist}(J, \operatorname{supp}[\mu])}{1+\operatorname{dist}(J, \operatorname{supp}[\mu])}=c_{0},
$$

say, where $c_{0}>0$. By the weak convergence,

$$
\begin{aligned}
0 & \leq c_{0} \limsup _{n \rightarrow \infty} \int_{J} \frac{1}{\left|E_{n, a}\right|^{2}} \leq \limsup _{n \rightarrow \infty} \int_{J} \frac{f}{\left|E_{n, a}\right|^{2}} \\
& \leq \lim _{n \rightarrow \infty} \int_{-\infty}^{\infty} \frac{f}{\left|E_{n, a}\right|^{2}} \\
& =\int f d \mu=0 .
\end{aligned}
$$

Finally,

$$
\left|E_{n, a_{n}}(t)\right|^{2}=\frac{\pi\left|t-a_{n}\right|^{2}\left|K_{n}\left(t, \overline{a_{n}}\right)\right|^{2}}{\left|\operatorname{Im} a_{n}\right| K_{n}\left(a_{n}, \overline{a_{n}}\right)} \leq 4 \pi \frac{\left(t^{2}+\left|a_{n}\right|^{2}\right)\left|K_{n}\left(t, \overline{a_{n}}\right)\right|^{2}}{\left|\operatorname{Im} a_{n}\right| K_{n}\left(a_{n}, \overline{a_{n}}\right)} .
$$

(b) We can cover compact $J$ by finitely many open intervals, each at a positive distance from $\operatorname{supp}[\mu]$. It then suffices to prove the assertion for the closure of just one of these intervals. So we assume $J$ consists of a single bounded interval. Next, as $\operatorname{supp}[\mu]$ is compact, we may choose a set $K$ consisting of two intervals 
that contains $\operatorname{supp}[\mu]$ but is disjoint from $J$. We can then choose polynomials $P_{n}$ of degree $\leq n-1$ such that

$$
\left|P_{n}\right| \leq 1 \text { in } K
$$

but

$$
\liminf _{n \rightarrow \infty}\left(\inf _{J}\left|P_{n}\right|\right)^{1 / n}>r>1 .
$$

In the special case where $J=[-\alpha, \alpha]$ and $K=[-1,-\beta] \cup[\beta, 1]$ with $0<\alpha<\beta<1$, we can just choose

$$
P_{n}(x)=T_{\left[\frac{n-1}{2}\right]}\left(-1+2\left(\frac{x^{2}-\beta^{2}}{1-\beta^{2}}\right)\right) .
$$

Here $T_{m}$ is the usual Chebyshev polynomial for $[-1,1]$ and $\left[\frac{n-1}{2}\right]$ denotes the integer part of $\frac{n-1}{2}$. The general case can be reduced to this special case, by enlarging the intervals of $K$ so that they become symmetric about $J$ and then using a linear transformation.

Armed with the $\left\{P_{n}\right\}$ satisfying (3.1) and (3.2), we apply (1.3): for large enough $n$,

$$
r^{2 n} \int_{J} \frac{1}{\left|E_{n, a_{n}}\right|^{2}} \leq \int_{J} \frac{P_{n}^{2}}{\left|E_{n, a_{n}}\right|^{2}} \leq \int P_{n}^{2} d \mu \leq \int d \mu .
$$

Finally, for $t$ in the compact set $J$, we have that for some $C>0$ depending only on the compact set,

$$
\left|E_{n, a_{n}}(t)\right|^{2} \leq C \frac{\left(1+\left|a_{n}\right|^{2}\right)\left|K_{n}\left(t, \overline{a_{n}}\right)\right|^{2}}{\left|\operatorname{Im} a_{n}\right| K_{n}\left(a_{n}, \overline{a_{n}}\right)}
$$

where $C$ is independent of $n$ and $t$. Together with the previous estimate, this gives (1.6).

Proof of Theorem 1.3. The Markov-Stieltjes inequalities [2, p. 33] assert that

$$
\sum_{j: x_{j n}<x_{k n}} \lambda_{n}\left(x_{j n}\right) \leq \int_{-\infty}^{x_{k n}} d \mu \leq \sum_{j: x_{j n} \leq x_{k n}} \lambda_{n}\left(x_{j n}\right) .
$$

Recall here that $\left\{x_{j n}\right\}$ are the zeros of $p_{n}$ and $\lambda_{n}$ is the $n$th Christoffel function for $\mu$. Since the measure $\frac{d t}{\left|E_{n, a_{n}}(t)\right|^{2}}$ has the same first $2 n-2$ power moments as $d \mu$, it has the same first $n-1$ orthogonal polynomials as $d \mu$ and the same $n$th Christoffel function. Thus it has the same Gauss quadrature involving $\left\{x_{j n}\right\}$ as $d \mu$, and so has the same Markov-Stieltjes inequalities (even though the $\left\{x_{j n}\right\}$ come from $p_{n}$, and there is no orthogonal polynomial of degree $n$ for $\left.\frac{d t}{\left|E_{n, a_{n}}(t)\right|^{2}}\right)$. A cursory scan of the proof of Theorem 5.4 in [2, p. 32] verifies this. So

$$
\sum_{j: x_{j n}<x_{k n}} \lambda_{n}\left(x_{j n}\right) \leq \int_{-\infty}^{x_{k n}} \frac{d t}{\left|E_{n, a_{n}}(t)\right|^{2}} \leq \sum_{j: x_{j n} \leq x_{k n}} \lambda_{n}\left(x_{j n}\right) .
$$

Combining the last two inequalities, we see that

$$
\left|\int_{-\infty}^{x_{k n}}\left(\frac{d t}{\left|E_{n, a_{n}}(t)\right|^{2}}-d \mu(t)\right)\right| \leq \lambda_{n}\left(x_{k n}\right) .
$$


Then also, if $x \in\left(x_{k n}, x_{k-1, n}\right)$, we deduce that

$$
\begin{aligned}
& \left|\int_{-\infty}^{x}\left(\frac{d t}{\left|E_{n, a_{n}}(t)\right|^{2}}-d \mu(t)\right)\right| \\
& \quad \leq \lambda_{n}\left(x_{k n}\right)+\max \left\{\int_{x_{k n}}^{x_{k-1, n}} \frac{d t}{\left|E_{n, a_{n}}(t)\right|^{2}}, \int_{x_{k n}}^{x_{k-1, n}} d \mu(t)\right\} \\
& \quad \leq \lambda_{n}\left(x_{k n}\right)+\lambda_{n}\left(x_{k-1, n}\right)+\lambda_{n}\left(x_{k n}\right),
\end{aligned}
$$

again by using the Markov-Stieltjes inequalities above. Now let $c$ and $d$ lie in $\operatorname{supp}[\mu]$. As $\mu$ is determinate, both $c$ and $d$ attract zeros of $p_{n}$ [2, Theorem 2.4, p. 67], so we can find for large enough $n$ and all $x \in[c, d]$ an index $k$ such that $x \in\left(x_{k n}, x_{k-1, n}\right)$ and both of $x_{k n}, x_{k-1, n}$ lie in $[c-\varepsilon, d+\varepsilon]$. Then we obtain that for large enough $n$,

$$
\sup \left|\int_{-\infty}^{x}\left(\frac{d t}{\left|E_{n, a_{n}}(t)\right|^{2}}-d \mu(t)\right)\right| \leq 3 \sup _{[c-\varepsilon, d+\varepsilon]} \lambda_{n} .
$$

Proof of Theorem 1.4. Let us assume that $\operatorname{Im} a>0$ and $P$ is a polynomial of degree $\leq 2 n-2$. Then

$$
\int_{-\infty}^{\infty} \frac{P}{\left|E_{n, a}\right|^{2}}=\int_{-\infty}^{\infty} \frac{P(t)}{E_{n, a}(t) E_{n, a}^{*}(t)} d t
$$

Here $E_{n, a}(z)$ has all its zeros in the lower half plane. By contrast, $E_{n, a}^{*}(z)$ is a multiple of $L_{n}(a, z)$, which has all its zeros $\left\{z_{j}\right\}_{j=1}^{n}$ in the upper half plane. By hypothesis, they are simple. Moreover, as $|t| \rightarrow \infty$,

$$
\frac{P(t)}{E_{n, a}(t) E_{n, a}^{*}(t)}=O\left(t^{-2}\right) .
$$

We can then use the residue theorem to deduce that

$$
\int_{-\infty}^{\infty} \frac{P(t)}{E_{n, a}(t) E_{n, a}^{*}(t)} d t=2 \pi i \sum_{j=1}^{n} \frac{P\left(z_{j}\right)}{E_{n, a}\left(z_{j}\right) E_{n, a}^{* \prime}\left(z_{j}\right)} .
$$

\section{ACKNOWLEDGEMENT}

Vili Totik provided useful references and perspectives during the OPSFA conference in Leuven in July 2009.

\section{REFERENCES}

[1] L. de Branges, Hilbert Spaces of Entire Functions, Prentice Hall, Upper Saddle River, New Jersey, 1968. MR0229011 (37:4590)

[2] G. Freud, Orthogonal Polynomials, Pergamon Press/Akademiai Kiado, Budapest, 1971.

[3] D. Krutikov and C. Remling, Schrödinger operators with sparse potentials: asymptotics of the Fourier transform of the spectral measure, Comm. Math. Phys. 223 (2001), 509-532. MR.1866165 (2002k:81064)

[4] D. S. Lubinsky, Universality limits for random matrices and de Branges spaces of entire functions, Journal of Functional Analysis 256 (2009), 3688-3729. MR2514057 
[5] B. Simon, Orthogonal Polynomials on the Unit Circle, Parts 1 and 2, American Mathematical Society, Providence, RI, 2005. MR2105088 (2006a:42002a)

[6] B. Simon, Orthogonal polynomials with exponentially decaying recursion coefficients, in Probability and Mathematical Physics (eds. D. Dawson, V. Jaksic and B. Vainberg), CRM Proc. and Lecture Notes, 42, American Mathematical Society, Providence, RI, 2007, 453-463. MR2352283(2008k:42087)

School of Mathematics, Georgia Institute of Technology, Atlanta, Georgia 30332 0160

E-mail address: lubinsky@math.gatech.edu 